\title{
An $\mathrm{OH}$ maser flare with a strong magnetic field in W75N
}

\author{
V. I. Slysh ${ }^{1}$ and V. Migenes ${ }^{2}$ \\ ${ }^{1}$ Astro Space Center, Lebedev Physical Institute, 84/32 Profsoyuznaya, Moscow, 117997 \\ email: vslsysh@asc.rssi.ru \\ ${ }^{2}$ Department of Astronomy, University of Guanajuato, P.O. Box 144, Guanajuato, GTO. \\ Mexico 36000, \\ email: vmigenes@astro.ugto.mx
}

\begin{abstract}
A flare of $\mathrm{OH}$ maser emission was discovered in W75N in 2000. Its location was determined with the VLBA to be within $110 \mathrm{AU}$ from one of the ultra-compact HII regions, VLA2. The flare consisted of several maser spots. Four of the spots were found to form Zeeman pairs, all of them with a magnetic field strength of about $40 \mathrm{mG}$. This is the highest ever magnetic field strength found in $\mathrm{OH}$ masers, an order of magnitude higher than in typical $\mathrm{OH}$ masers. We discuss the possible source for the enhanced magnetic field and its relation to the flare event.
\end{abstract}

Keywords. ISM:magnetic fields, stars: flare, maser

\section{Observations}

The new observations of the $\mathrm{OH}$ maser emission in W75N were conducted on 2001 January 01 with the VLBA in a snapshot mode of 6 -min duration. The velocity resolution was $0.176 \mathrm{~km} \mathrm{~s}^{-1}$ covering $45 \mathrm{~km} \mathrm{~s}^{-1}$ in each of the $\mathrm{OH}$ main lines at $1665 \mathrm{MHz}$ and $1667 \mathrm{MHz}$. In addition, we reduced and analyzed archived observations from the EVN and VLBA for 2000 September 27 and 2000 November 22 and 2001 January 6, respectively. Both data sets had the same velocity resolution though different velocity coverage.

All three sets of data have been obtained during the maximum phase of the $\mathrm{OH}$ maser flare. The data were reduced in the standard way using NRAO AIPS package. Most of the maser spots were unresolved by the synthesized beam. The absolute position given in Table 1 was measured through fringe rates using AIPS task FRMAP.

\section{Discussion: Flare and magnetic field}

Two new strong spectral features have appeared since the 1998 observations, at the low-velocity side of the spectrum. On the other hand, two relatively strong spectral features from the 1998 spectrum became a factor of three weaker in the 2000-2001 spectra. It is also evident from the spectra that the new "flare" features were rapidly evolving in about a three months time interval, between 2000 September 27 (EVN) and 2001 January 1 (VLBA). Four months later, on 2001 April 12, these features had become considerably weaker as observed with the Bear Lake 64-m single dish telescope (Alakoz et al. 2005). In the same time interval the rest of the spectral features remained unchanged. All constant features are connected with the ultra-compact HII region VLA1 while the variable features are connected with VLA2.

Compared to the 1998 map (Slysh et al. 2002) several additional spots have been detected, partly due to a higher sensitivity of the new observations. The other features 
Table 1. OH Zeeman pairs in W75N

\begin{tabular}{lrrrrrrrrr}
\hline $\begin{array}{l}\text { Zeeman } \\
\text { Pairs }\end{array}$ & $\begin{array}{r}\Delta \text { RA } \\
\text { RCP }\end{array}$ & $\begin{array}{r}\Delta \text { DEC } \\
\text { RCP }\end{array}$ & $\begin{array}{r}\text { Velocity } \\
(\mathrm{km} / \mathrm{s})\end{array}$ & $\begin{array}{r}\Delta \mathrm{RA} \\
\text { LCP }\end{array}$ & $\begin{array}{r}\Delta \mathrm{DEC} \\
\text { LCP }\end{array}$ & $\begin{array}{r}\text { Velocity } \\
(\mathrm{km} / \mathrm{s})\end{array}$ & $\begin{array}{c}\text { B-field } \\
\text { Strength } \\
(\mathrm{mG})\end{array}$ & $\begin{array}{c}\text { Separation } \\
(\mathrm{mas})\end{array}$ & $\begin{array}{c}\text { Associate } \\
\text { HII Region }\end{array}$ \\
\hline Z1 $^{a}$ & 0.0 & 0.0 & 12.45 & 0.16 & 0.03 & 9.28 & 5.4 & 0.2 & VLA1 \\
Z2 $_{\text {Z3 }}$ & 205.42 & 666.92 & 8.07 & 206.91 & 668.90 & 5.43 & $7.5^{b}$ & 2.5 & VLA1 \\
Z4 & 788.80 & 1345.93 & 7.24 & 489.13 & 1345.29 & 5.13 & 3.6 & 0.7 & VLA1 \\
Z5 & 765.77 & -178.12 & 24.40 & 766.15 & -177.75 & -0.48 & 42.2 & 1.6 & VLA2 \\
Z6 & 770.30 & -135.24 & 19.80 & 767.59 & -132.00 & 4.76 & $42.5^{c}$ & 3.5 & VLA2 \\
Z7 & 770.41 & -129.98 & 29.27 & 770.89 & -125.00 & 4.71 & $41.6^{d}$ & 2.7 & VLA2 \\
\hline
\end{tabular}

${ }^{a} \mathrm{Z} 1$ is spot $\mathrm{A}$ : with a measured absolute position of $\mathrm{RA}=20 \mathrm{~h} 38 \mathrm{~m} 36 \mathrm{~s} .416$; DEC $=4237^{\prime} 34$ ". 42 (0".01) (J2000). ${ }^{b} 1667 \mathrm{MHz} .{ }^{c} 1667 \mathrm{MHz}$ (Fish et al. 2005, table 15). This Zeeman pair was probably overlooked by the authors, or dismissed as showing too large a velocity separation.

${ }^{d}$ From EVN data.

are really new as they are related to the flare which took place between 1998 and 2000 . Also, more accurate absolute positions of $\mathrm{OH}$ spots were obtained and are given in Table 1, as well as the positions of the $1667 \mathrm{MHz}$ spots relative to the $1665 \mathrm{MHz}$ spots.

$\mathrm{H}_{2} \mathrm{O}$-masers have also been found near the $\mathrm{OH}$ masers in $\mathrm{W} 75 \mathrm{~N}$, located in two clusters around VLA1 and VLA2. Torrelles et al. (2003) have found a shell of water masers around the ultra-compact HII region VLA2 with a radius of 160 AU. The shell is expanding with a velocity of $28 \mathrm{~km} \mathrm{~s}^{-1}$, perhaps episodically as in a recurrent outflow. The high magnetic field $\mathrm{OH}$ maser spots Z4-Z7 are located very close to VLA2, at a distance of 55 mas ( \pm 40 mas), or at the projected distance of $110 \mathrm{AU}( \pm 80 \mathrm{AU})$. Therefore, the $\mathrm{OH}$ masers may well be located in the same shell as the water masers. The magnetic field in water masers associated with star-forming regions is typically around $100 \mathrm{mG}$, which is about the same order of magnitude as in the $\mathrm{OH}$ maser flare reported here.

The appearance of new strong maser features and the simultaneous dimming of nearby features can be interpreted as originating from the passage of a magnetohydrodynamic (MHD) shock (Alakoz et al. 2005). The shock was probably generated by the exciting star of VLA2 and propagated in the gas of the stellar wind.

\section{Conclusions}

A very strong magnetic field of $40 \mathrm{mG}$ has been detected in several $\mathrm{OH}$ masers spots which have appeared during a flare of $\mathrm{OH}$ maser emission in 2000, within $110 \mathrm{AU}$ from the ultra-compact HII region. The magnetic field probably originates in the exciting star where its intensity is about $500 \mathrm{G}$, or from the compression of interstellar gas by MHD shock, or in icy planetary bodies serving as nuclei for the maser spot emission. More frequent high angular resolution observations of future flares may help to distinguish between these models.

\section{References}

Alakoz A. V., Slysh V. I., Popov M. V. \& Val'tts I. E., 2005, Astron. Letters 31, 375

Fish V. L., Reid M. J., Argon A. L. \& Zheng X.-W., 2005, ApJS 160, 220

Slysh V. I., Migenes V., Val'tts I. E., Lyubchenko S. Yu., Horiuchi S., Altunin V. I., Fomalont

E. B. \& Inoue M., 2002, ApJ 564, 317

Torrelles J. M. et al., 2003, ApJL 598, L115 